\title{
$\simeq$ Praxisnahes Lernen in der Fachweiterbildung Anästhesie- und Intensivpflege*
}

\author{
Angela Boonen ${ }^{1}$, Ingrid Darmann ${ }^{2}$ \\ ${ }^{1}$ Bildungszentrum des Universitätsklinikums Hamburg-Eppendorf, Hamburg \\ 2 Fachbereich Human- und Gesundheitswissenschaften, Universität Bremen
}

\begin{abstract}
Zusammenfassung: In Abgrenzung von einer einseitig technik- und medizinorientierten Ausrichtung der Fachweiterbildung Anästhesie- und Intensivpflege wird in diesem Beitrag ein fächerintegratives Curriculum vorgestellt, in dessen Mittelpunkt typische Pflegesituationen stehen, anhand derer klinische Urteilsfähigkeit und prozessorientierte Problemlösungskompetenzen gefördert werden. Die traditionellen Lehr-/Lernformen des Dozentenunterrichts und des klinischen Unterrichts wurden mit dem Ziel einer stärkeren Pflegesituations- und Pflegeprozessorientierung weiterentwickelt. Außerdem wurde ein nicht unerheblicher Teil des Dozentenunterrichts ersetzt durch Problemorientiertes Lernen (POL) in Kleingruppen. Die Evaluation stellte sowohl Effekte der getroffenen Veränderungen als auch Skepsis auf Seiten der Teilnehmer fest.
\end{abstract}

\section{Einleitung}

Praxisnahes Lernen steht im Mittelpunkt der curricularen Reformen in der Fachweiterbildung Anästhesie- und Intensivpflege am Universitätsklinikum

Angela Boonen ist Lehrerin für Pflegeberufe und leitet die Fachweiterbildung Anästhesie- und Intensivpflege am Universitätsklinikum Hamburg-Eppendorf. Prof. Dr. Ingrid Darmann ist Professorin am Fachbereich Human- und Gesundheitswissenschaften der Universität Bremen für Pflegewissenschaft mit dem Schwerpunkt pflegetherapeutische Grundlagen und ihre pflegedidaktische Vermittlung.

intensiv 2004; 12: 290-296

(c) Georg Thieme Verlag KG Stuttgart · New York ISSN 0942-6035

DOI 10.1055/s-2004-813572
Hamburg-Eppendorf (UKE). Der Anlass für die Reformen war die Modifizierung der Fortbildungs- und Prüfungsordnung zur Fachkrankenschwester/zum Fachkrankenpfleger für Intensivpflege und Anästhesie im Jahr 2000 [1] sowie die Kritik an der bisherigen Form der Fachweiterbildung. Zurzeit wird ein fächerintegratives Curriculum basierend auf der Methode des Problemorientierten Lernens (POL) entwickelt, implementiert und evaluiert.

Durchführung und wissenschafliche Evaluation werden finanziell durch die Robert-Bosch-Stiftung unterstützt. In diesem Beitrag werden die Ziele sowie die getroffenen Veränderungen (Curriculumentwicklung, klinischer Unterricht, Problemorientiertes Lernen) zunächst aus Sicht der Leiterin der Fachweiterbildung (Angela Boonen) beschrieben, aus Sicht der Evaluatorin (Ingrid Darmann) wird anschließend jeweils der theoretische Hintergrund erschlossen. Abschließend werden erste Ergebnisse der Evaluation dargestellt.

\section{Ziele der Fachweiterbildung}

\section{Aus Sicht der Fachweiterbildung}

Die Fachweiterbildung Anästhesie- und Intensivpflege soll die Teilnehmer für die Tätigkeit im Intensivbereich und in der Anästhesie qualifizieren und ihnen zur Erfüllung dieser Aufgaben die notwendigen speziellen Kenntnisse, Fertigkeiten und Verhaltensweisen auf der Grundlage eines engen Theorie-PraxisTransfers vermitteln. Die Pflege in der Anästhesie und auf der Intensivstation ist dadurch geprägt, dass zum einen die

\footnotetext{
Überarbeitete Fassung eines Vortrags, gehalten auf dem 14. Symposium für Intensivmedizin und Intensivpflege in Bremen am 19. Februar 2004.
}

dort behandelten Menschen hochgradig abhängig sind und zum anderen jederzeit mit Notfällen oder akut lebensbedrohlichen Situationen gerechnet werden muss. Beide Bereiche zeichnen sich dadurch aus, dass die Vitalfunktionen der dort behandelten Patienten von einer Vielzahl an Geräten überwacht und unterstützt werden. Diese Geräte müssen von den Pflegenden sicher beherrscht und sollten als Unterstützung und nicht als zentrale Einheit der Arbeit mit den Patienten gesehen werden. Neben diesen Gemeinsamkeiten unterscheidet sich die Arbeit in der Anästhesie grundlegend von der auf den Intensivstationen. Die Tätigkeit in der Anästhesie ist durch die enge $\mathrm{Zu}$ sammenarbeit mit den Anästhesisten geprägt. Standardisierte Arbeitsabläufe ermöglichen einen reibungslosen $\mathrm{Ab}$ lauf. Die Kommunikation und die Absprache mit den anderen Berufsgruppen stehen im Mittelpunkt der Tätigkeit in der Anästhesie. Patientenorientierte Pflege findet aufgrund des nur kurzen Kontaktes zwischen Pflegekraft und Patient nur bedingt statt. Die Pflege auf den Intensivstationen ist dagegen nicht so stark standardisiert und erfordert einen situativen Planungsprozess entlang des Pflegeprozessmodells. Die Kommunikation mit den Patienten ist häufig erschwert und es ist die Aufgabe des Pflegepersonals, die Patienten mit all ihren Problemen und Bedürfnissen als individuelle Persönlichkeiten in den Pflegeprozess einzubeziehen. Hieraus resultieren hohe Ansprüche an die fachlich-technische, soziale und personale Kompetenz der dort tätigen Pflegekräfte.

In der Vergangenheit lag der Schwerpunkt der Weiterbildung auf der Vermittlung medizinischer Inhalte. Ziel der neu strukturierten Fachweiterbildung am Universitätsklinikum Hamburg-Eppendorf ist es, den Teilnehmern ein pflegeorientiertes Berufsverständnis zu vermitteln. Zwar sollten die Pflegenden über ein fundiertes medizinisches Wissen verfügen, dieses sollte jedoch nicht im Zentrum ihres Denkens stehen. Vielmehr sollten sie auf der Basis dieses Wissens in der Lage sein, pflegerische Probleme zu erkennen, zu deuten und die entsprechenden Maßnahmen einzuleiten, also pflegeprozessorientiert zu arbeiten. Zudem gilt es, die Pflege auf den Intensivstationen und in der Anästhesieabteilung auch unter knapperen Ressourcen professionell zu gestalten. Um diesen zunehmenden Anforderun- 
gen im Alltag gewachsen zu sein, müssen sich die Schwerpunkte der Fachweiterbildung verändern. In der heutigen Zeit muss es unter anderem darum gehen, neben der Vermittlung von Faktenwissen die Teilnehmer zu befähigen, sich neues und erweitertes Wissen selbstständig zu erarbeiten. Diese Fähigkeit wird künftig noch wichtiger sein, da es vermutlich verstärkt den Fachdisziplinen übergeordnete Intensivstationen geben und sich dadurch das Spektrum der Krankheitsbilder auf den Intensivstationen vergrößern wird. Dies erfordert von den Pflegenden eine höhere Flexibilität. Sie müssen in der Lage sein, sich schnell auf eine neue Situation einzustellen und einmal erworbene Erkenntnisse auf neue Situationen zu übertragen. Des Weiteren sollten sich Pflegende in Kooperation mit anderen Berufsgruppen argumentativ auseinander setzen können und sie sollten in der Lage sein, ihr Handeln vor dem Hintergrund neuer Erkenntnisse zu überprüfen. Aus diesem Grund sollte die Reflexion des eigenen Handelns im Rahmen der Fachweiterbildung implementiert werden. Die Fähigkeit der Übertragung der erworbenen Erkenntnisse auf eine neue Situation führt langfristig zu einer Steigerung der Handlungsfähigkeit.

\section{Aus Sicht der Evaluation}

Wenn von Pflegeorientierung der Fachweiterbildung Anästhesie- und Intensivpflege gesprochen wird, so ist damit nicht allein eine stärkere Gewichtung von sozialen und kommunikativen Kompetenzen gemeint, vielmehr sollen Pflegephänomene, Pflegeprobleme oder noch umfassender: Pflegesituationen und deren Gestaltung durch beruflich Pflegende im Mittelpunkt der Weiterbildung stehen. Das in der Intensivpflege zweifellos unabdingbare Wissen z.B. über die Pathophysiologie von Erkrankungen, über die Wirkungen von Arzneimitteln oder die Funktionsweise von Maschinen wird in seinem Beitrag zum Verständnis und zur Gestaltung von Pflegesituationen relevant und nicht als Selbstzweck. Professionelle Pflege oder Pflegeexpertise [2] zeigt sich darin, dass Pflegende in komplexen und einzigartigen Pflegesituationen schnell und situationsangemessen handeln. Dabei kommt als Fähigkeit situative Urteilskraft bzw. die diagnostische Kompetenz zum Ausdruck, den Sinn einer Situation anhand der wahrgenommenen Konfigu- ration angemessen $\mathrm{zu}$ rekonstruieren und daraus Schlussfolgerungen für Handlungsoptionen zu ziehen. Ziel der curricularen Reformen der Fachweiterbildung mit der Perspektive Pflegeorientierung sind somit die Verbesserung und Erweiterung der Urteilsund Handlungsfähigkeit der Teilnehmer in konkreten Pflegesituationen.

Will man situative Urteils- und Handlungsfähigkeit fördern, muss man sich darüber klar werden, was man unter einem guten situativen Urteil verstehen will. Aus Sicht des Konstruktivismus und des Interaktionismus handeln wir stets auf der Grundlage von Bedeutungen, die wir Dingen und Interaktionen zuweisen, d.h. unser Verhalten ist Ergebnis von subjektiven Deutungsprozessen und kann nicht zwingend aus einer bestimmten situativen Konstellation abgeleitet werden [3]. Ob wir mit unserem Urteil richtig liegen oder nicht, hierfür gibt es keinen objektiven Maßstab. Für die Pflegekraft mag es zufriedenstellend sein, wenn ein zuvor unruhiger Patient nach Valiuminjektion ruhig ist, aus Sicht des Patienten, dessen die Unruhe verursachendes Problem nicht behoben wurde, aber nicht. Ein gutes situatives Urteil ist daher erstens eines, dass in dem Bewusstsein getroffen wurde, dass Urteile perspektivisch und daher stets überprüfungsbedürftig sind. Zweitens sollte sich das situative Urteil professionell Pflegender (im Unterschied etwa zur Laienpflege) prinzipiell auf eine überprüfbare und überprüfte Wissens- und Kenntnisbasis beziehen [4]. Zwar werden Entscheidungen häufig unter Handlungsdruck getroffen, die Begründungsverpflichtung wird deswegen aber nicht aufgehoben, sondern nur aufgeschoben (ebd.). Ein Nachholen der Begründungsverpflichtung kann etwa durch Evaluation der Handlungswirkungen oder durch Reflexion der gefundenen Lösung erfolgen. Der Erwerb neuen Wissens dient aus dieser Perspektive der Erweiterung von Deutungs- und Handlungsmöglichkeiten sowie der Überprüfung der eigenen Handlungsbegründungen. Der Theorie-Praxis-Transfer erfolgt nicht durch technokratische Anwendung von Handlungsregeln, sondern reflexiv durch die multiperspektivische Deutung von Pflegesituationen.

Vielen beruflich Pflegenden und den meisten Teilnehmern der Fachweiterbildung ist der Prozess der Urteilsbil- dung gar nicht bewusst. Dies hängt zum einen damit zusammen, dass zum Zeitpunkt des Urteilens die Aufmerksamkeit nicht auf das Urteilen, sondern auf die Situation gerichtet ist. Das Urteil erfolgt intuitiv, ihm ist das Wissen über die der Entscheidung zugrunde liegenden Begründungen implizit [5]. Zum anderen ist die mangelnde Bewusstheit aber auch ein Symptom für das mechanistische Pflegeverständnis der meisten beruflich Pflegenden, was sich etwa in der Auffassung ausdrückt, es gäbe für eine bestimmte Pflegesituation eine einzig „wahre“ Bedeutung und eine einzig zutreffende Reaktion. Viele Pflegende erkennen weder die Deutungsoffenheit noch die Vielfalt an Handlungsoptionen, die prinzipiell in jeder Situation gegeben sind [6]. Wissen wird unter dem mechanistischen Verständnis als Handlungsanweisung für die Praxis betrachtet. Diese reduktionistische Sichtweise hat schwerwiegende negative Folgen. Sie führt zu einer mangelnden Kritikfähigkeit gegenüber den eigenen Deutungen, zu „impliziten Tunnelperspektiven“ [7] und damit zu Erstarrung und Routinisierung der pflegerischen Handlungen. In der Folge werden für individuelle Problemlagen der Patienten, z.B. Atemnot oder Desorientiertheit, ohne Berücksichtigung der in dieser einzigartigen Situation möglichen Hintergründe standardisierte Lösungen praktiziert.

Zusammenfassend hat die Fachweiterbildung Anästhesie- und Intensivpflege die Aufgabe, den Teilnehmern den Prozess der Konstruktion von Bedeutungen (also der Urteilsbildung) bewusst $\mathrm{zu}$ machen, die Teilnehmer zur Reflexion und ggf. Korrektur ihrer unter Handlungsdruck gewonnenen routinisierten Lösungen anzuregen sowie ihren Interpretations- und Begründungsrahmen durch neues Wissen zu erweitern. Der Theorie-Praxis-Transfer lässt sich vor diesem Hintergrund als komplexer Transformations- und Reflexionsprozess konzipieren [8]. Natürlich kann es in der Fachweiterbildung Anästhesieund Intensivpflege nicht ausschließlich um Reflexivität gehen, auch die Vermittlung von Regelwissen und Handlungssicherheit ist von zentraler Bedeutung. Diese Sicherheiten sind aber immer wieder zugunsten der Reflexivität aufzubrechen. 


\section{Curriculumentwicklung}

\section{Aus Sicht der Fachweiterbildung}

Mit dem Curriculum wird beabsichtigt, die bisherige wissenschaftsorientierte Fächerstruktur zumindest teilweise aufzubrechen und eine neue Systematik in den Mittelpunkt zu stellen [9]. Strukturierendes Element sind typische, komplexe Pflegesituationen aus der Praxis im Anästhesie- und Intensivbereich.

Bisher wurden u.a. folgende Pflegesituationen ermittelt:

- Pflegesituationen von Patienten mit beeinträchtigter Atmung;

- Pflegesituationen von wahrnehmungsbeeinträchtigten Patienten;

- Pflegesituationen von Herz-Kreislaufbeeinträchtigten Patienten;

- Pflegsituationen von Patienten in der Anästhesie.

Drei verschiedene Lehr-/Lernformen werden zur Kompetenzentwicklung im Rahmen der Pflegesituationen eingesetzt, nämlich der Dozentenunterricht, das Problemorientierte Lernen und der klinische Unterricht. Unter Dozentenunterricht verstehen wir den Unterricht im Kursverband. Ergänzend zum Dozentenunterricht findet das Problemorientierte Lernen (POL) in Kleingruppen statt. Das Lernen in Praxissituationen erfolgt auf sieben verschiedenen Intensivstationen und in der Anästhesieabteilung, die elf operative Kliniken versorgt. Im Rahmen der Einsätze erhalten die Teilnehmer regelmäßige klinische Unterrichte in Form von individuellem Einzelunterricht. Alle drei Elemente (Dozentenunterricht, POL, klinischer Unterricht) stehen in engem Zusammenhang.

In den Dozentenunterrichten werden aus Sicht verschiedener Fachdisziplinen Teilaspekte zu diesen Pflegesituationen dargestellt und einige Inhalte werden fächerintegrativ im Team-teaching (Pflegende - Mediziner - Lehrer) angeboten.

\section{Aus Sicht der Evaluation}

Indem das Curriculum anhand von Pflegesituationen strukturiert ist, lässt es sich dem situationsorientierten Ansatz der Curriculumentwicklung [10] zuordnen und entspricht damit dem aktuellen curricularen Trend in der Berufsund Wirtschaftspädagogik, der in den so genannten lernfeldorientierten Lehrplänen seinen Niederschlag findet [11]. Durch die situationsorientierte Strukturierung soll sichergestellt werden, dass die Ziele und Inhalte der Fachweiterbildung den tatsächlichen Anforderungen der Berufspraxis entsprechen, und es werden günstigere Rahmenbedingungen für handlungsorientierte Lehr-/ Lernformen und damit für den Erwerb von transferfähigem Wissen und Können geschaffen.

Das Curriculum für die Fachweiterbildung Anästhesie- und Intensivpflege befindet sich zurzeit noch in der Entwicklung. Zwei Aufgaben sind zukünftig vorrangig zu bearbeiten. Erstens ist es erforderlich, typische Pflegesituationen mittels empirischer Untersuchungen und nicht allein pragmatisch zu identifizieren. Zweitens sind die Unterrichtsgegenstände im Hinblick auf ihren bildenden Gehalt zu befragen und auszuwählen, also im Hinblick darauf, welche allgemeinen Zusammenhänge, Widersprüche, Erkenntnisse und Fähigkeiten sich anhand dieses Gegenstandes aneignen oder vermitteln lassen [12].

\section{Klinischer Unterricht}

\section{Aus Sicht der Fachweiterbildung}

Die Fortbildungs- und Prüfungsordnung sieht 1580 Stunden berufspraktische Anteile vor, von denen mindestens 10\% (158 Stunden) unter Anleitung einer Mentorin mit pädagogischer Qualifikation durchgeführt werden müssen. Der klinische Unterricht findet als individueller Einzelunterricht auf den Intensivstationen und in der Anästhesieabteilung statt und wird von Praxisanleiterinnen, die der Lehrgangsleitung fachlich unterstellt sind, durchgeführt. Mit den klinischen Unterrichten soll die Problemlösungsfähigkeit in Alltagssituationen gefördert werden, um langfristig die Handlungskompetenz in beruflichen Problemsituationen zu steigern.

Die Praxisanleiterinnen wurden von Anfang an in die Curriculumentwicklung einbezogen, da sie eine wichtige Schnittstelle zwischen Theorie und Praxis bilden und um ihnen den Zusammenhang zwischen den schulischen und den klinischen Unterrichten zu verdeutlichen. $\mathrm{Zu}$ Beginn der gemeinsamen Arbeit wurden übergeordnete
Inhalte der klinischen Unterrichte anhand der bereits erwähnten Pflegesituationen in einem praktischen Weiterbildungsordner zusammengestellt. Der Ordner bietet eine Übersicht über die zu erlernenden Themen. Außerdem werden darin die schriftlichen Rückmeldungen, die zu den klinischen Unterrichten von den Praxisanleiterinnen erstellt werden, abgeheftet. Der Ordner bietet nicht nur den Teilnehmern, sondern auch den Praxisanleiterinnen, den Mentoren der Stationen und der Lehrgangsleitung einen kontinuierlichen Einblick in die individuelle Entwicklung jedes Einzelnen.

Über den Einbezug der Praxisanleiterinnen in die Curriculumentwicklung und in die Dozentenunterrichte ist es gelungen, dass sich die Praxisanleiterinnen für eine möglichst zeitnahe Umsetzung des in „der Theorie“ erlernten Wissens in den klinischen Unterrichten bemühen, und es kann gewährleistet werden, dass die Teilnehmer eine vergleichbare Anzahl an klinischen Unterrichten erhalten.

Trotz dieser Fortschritte fällt z.B. in Prüfungen und in den POL-Sitzungen auf, dass die Teilnehmer weiterhin Schwierigkeiten haben, ihr theoretisches Wissen auf (reale, klinische) Pflegesituationen zu transferieren. Der Wunsch nach Rezepten ist hoch und es entsteht der Eindruck, dass wie zuvor immer noch in erster Linie für Prüfungen gelernt wird und das Verständnis für den eigenen Nutzen fehlt. Von Seiten der Evaluatorin wurde beanstandet, dass die klinischen Unterrichte vor allem auf die motorisch-praktische Durchführung von Tätigkeiten und nicht auf die Urteils- und Handlungsfähigkeit in komplexen Situationen abzielen. Daher sollen zukünftig die klinischen Unterrichte stärker anhand der Schritte des Pflegeprozesses strukturiert werden. Des Weiteren sollen die Lernenden von den Praxisanleiterinnen vermehrt aufgefordert werden, erworbenes Wissen zur Situationsdeutung heranzuziehen.

\section{Aus Sicht der Evaluation}

Üblicherweise besteht ein zentrales Ziel von klinischem Unterricht in der Vervollkommnung des Handlings. Zwar sollte dieses Ziel nicht vernachlässigt werden, der klinische Unterricht darf aber nicht dabei stehenbleiben, sondern 
sollte auch Wahrnehmungs-, Urteilsund Handlungsfähigkeit in komplexen Pflegesituationen anbahnen.

Nach dem Cognitive-ApprenticeshipAnsatz kann die Expertise der situativen Diagnostik an die Lernenden weitergegeben werden, indem Experten (in diesem Fall Praxisanleiterinnen) die geeignete Vorgehensweise vormachen und dabei zugleich ihre kognitiven Strategien und Prozesse bei der Situationserfassung verbalisieren [13, 14]. Als Hilfsinstrument für die Situationserfassung können hierfür die Schritte des Pflegeprozesses herangezogen werden. Abweichend von den klassischen Formen des cognitive apprenticeship und stärker orientiert an konstruktivistischen Grundsätzen wurden in empirischen Untersuchungen noch bessere Effekte festgestellt, wenn die Lernenden zunächst selbst das Problem bearbeiten und erst dann das Modell/den Experten beobachten [15]. Dies entspricht auch dem hier verfolgten Anliegen, die Teilnehmer der Fachweiterbildung zur Reflexion ihrer möglicherweise bereits erstarrten Deutungsschemata anzuregen. Zwar hat der Lehrende in diesem Ansatz zumindest anfangs eine stark lehrerzentrierte Position inne, diese soll aber durch ein kognitive Prozesse anregendes Lehrgespräch ausgefüllt werden [16]. Für die Fachweiterbildung kann das Lehrgespräch zwischen Lernenden und Experten etwa die Situationsdiagnose, die Begründung eines Urteils, die Konfrontation mit anderen Urteilen (z.B. des Urteils der Praxisanleiterin) oder den Vergleich mit anderen ähnlichen oder unähnlichen Pflegesituationen aufgreifen. Weil der Prozess der Urteilsbildung aber nur bedingt durch Regeln beschreibbar ist, können die Strategien der Urteilsbildung und der Handlungsgenerierung durch Verbalisierung nur teilweise an die Lernenden weitergegeben werden. Der verbleibende Rest, der sich nicht explizieren lässt, ist von den Teilnehmern durch das Einfühlen in „das Geschick des Experten“ [17] zu erschließen. Dies setzt voraus, dass die Teilnehmer den Praxisanleiterinnen das Vertrauen entgegenbringen, dass sie von ihnen auch tatsächlich etwas lernen können.

\section{Problemorientiertes Lernen}

\section{Aus Sicht der Fachweiterbildung}

Das Problemorientierte Lernen (POL) [18, 19, 20, 21] ist ein didaktisches Vorgehen zur gezielten Erarbeitung von Lerninhalten in Kleingruppen (max. 8 Teilnehmer). Es besteht aus einer Kombination von Kleingruppendiskussionen und Selbststudium. Die Kleingruppenarbeit findet dabei unter Anleitung eines Moderators statt. Der Ausgangspunkt für das POL ist eine Problemstellung oder ein Fallbeispiel aus der täglichen Berufspraxis der Teilnehmer. Der Ablauf ist in Lernschritte gegliedert (Siebensprung): In Schritt 1-5 werden die Fall- bzw. Problemstrukturen analysiert und Lernziele formuliert, in Schritt 6 erfolgt die selbstständige Wissensaneignung und in Schritt 7 das Zusammentragen der Ergebnisse. Am Ende einer Problembearbeitung kann - je nach Ausbildungsstand - deren Lösung stehen. Entscheidend für das POL ist nicht das Ergebnis der Problembearbeitung, die Problemlösung, sondern der Weg zu ihr, die Strategie.

Die Fachweiterbildung Anästhesie- und Intensivpflege umfasst laut Fortbildungs- und Prüfungsordnung insgesamt 720 Stunden theoretischen Unterricht. Zurzeit nimmt das POL im Rahmen 156 von 421 Pflegestunden (entspricht 37\%) ein (vgl. Abb.1). Im Durchschnitt bearbeiten die Teilnehmer pro Monat einen Fall, dies entspricht 19 Fällen in 2 Jahren. Die erste Sitzung (Schritt 1-5) erstreckt sich über zwei Unterrichtsstunden (90 Min.), im Anschluss daran erhalten die Teilnehmer vier Unterrichtsstunden Studienzeit zur Bearbeitung der Lernziele (Schritt 6) und in der nächsten Woche findet die zweite Sitzung, ebenfalls im Rahmen von zwei Unterrichtsstunden statt (Schritt 7). Moderiert wurden die Sitzungen bislang von geschulten Moderatoren (die bereits erwähnten Praxisanleiterinnen). Das POL hat eine fächerintegrative Funktion, da die Fälle sowohl pflegerische als auch medizinische Inhalte enthalten und sich die Teilnehmer im Rahmen der Lernzielbearbeitung mit verschiedenen Schwerpunktbereichen auseinander setzen.

Beim Problemorientierten Lernen eignen sich die Teilnehmer Fachwissen und die methodische Kompetenz zur Quellensuche und -kritik an, sie verbessern ihre Problemlösungsfähigkeiten, erweitern ihr Handlungsrepertoire und hinterfragen routinisierte Handlungspraktiken. Neben der Fach- und der Methodenkompetenz werden aber auch kommunikative Kompetenz und Teamfähigkeit gefördert.

Nach Einführung des Problemorientierten Lernens machten sich die ersten Probleme an den Rahmenbedingungen (Lernort- und -zeit) fest. Die POL-Sitzungen fanden im Anschluss an den Frühdienst statt und den Teilnehmern fiel es schwer, sich auf die Arbeit in den POL-Gruppen zu konzentrieren. Die ursprünglich geplanten zusätzlichen variablen Studientage für die Erarbeitung der Lernziele wurden nicht mit dem POL in Verbindung gebracht und die Teilnehmer hatten subjektiv das Gefühl, die Erarbeitung der Lernziele komplett in ihrer „Freizeit“ bewältigen zu müssen. Des Weiteren stellte es sich als schwierig dar, geeignete, ungestörte Räume auf dem Klinikgelände zu finden. Die Akzeptanz des POL wurde an den schlechten Rahmenbedingungen

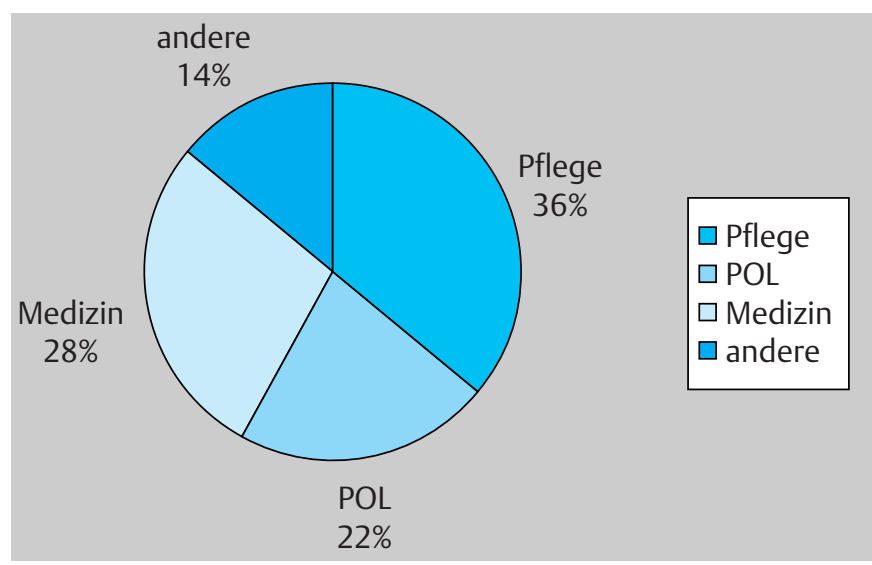

Abb. 1 Prozentualer Anteil des Problemorientierten Lernens am schulischen Unterricht. 
gemessen; dies bestätigten auch die ersten Ergebnisse der Evaluation [23]. Daraufhin integrierten wir die POLStunden in die Studientage und die Sitzungen finden seitdem im Bildungszentrum statt. Die Änderungen wurden von allen Teilnehmern sehr positiv aufgenommen.

Durch die Evaluation wurden außerdem Unzulänglichkeiten im Bereich der POL-Fälle und der Moderation ermittelt. Es wurde festgestellt, dass die Fälle nicht zur Ermittlung unterschiedlicher Deutungsmöglichkeiten und zur Entwicklung von Handlungsalternativen geeignet waren und dass sich die Teilnehmer in den POL-Gruppen mit oberflächlichem Wissen zufrieden gaben. Auf Empfehlung der Evalutorin werden die Fälle nun so konstruiert, dass sie ungelöste oder nicht befriedigend gelöste Problemsituationen beinhalten, die aus wechselnden Perspektiven betrachtet werden können. Die Moderation wurde dahingehend konkretisiert, dass die Probleme näher am Fall formuliert werden und dass die Moderatorinnen die Gruppen vermehrt anregen, Hypothesen/Vermutungen zu den Hintergründen der bestehenden Probleme zu bilden. Außerdem decken die Moderatorinnen durch gezieltes Nachfragen Wissenslücken auf bzw. fordern Begründungen zu Behauptungen ein. Auch die zweite Sitzung wurde deutlicher strukturiert, als dies bisher der Fall war.

\section{Aus Sicht der Evaluation}

In der Fachweiterbildung Anästhesieund Intensivpflege am UKE wird ein POL-Ansatz verfolgt, mit dem insbesondere der Erwerb von Deutungswissen und die Förderung hermeneutischen Fallverstehens angeregt werden soll. In den meisten Veröffentlichungen zum POL wird demgegenüber der Schwerpunkt auf die Problemlösung (z.B. im Zusammenhang mit dem Konzept des evidence-based nursing) [24, 25] oder auf den Erwerb von kontextbezogenem Wissen gelegt [26, 27, 27, 29]. Gemeinsam ist diesen Ansätzen, dass sie die Problemdefinition und -deutung vernachlässigen oder den Eindruck erwecken, die Deutung könne technizistisch durch Anwendung des „richtigen“ Wissens erfolgen. In der Durchführung kommt dies etwa darin zum Tragen, dass den Lernenden kontextualisierte
Krankheitsbilder präsentiert werden, für die es eine richtige Deutung gibt.

Um reflexive Deutungskompetenz zu fördern, wird in der Fachweiterbildung des UKE bei der Fallkonstruktion ein interaktionsorientiertes Pflegeverständnis zugrunde gelegt, wonach offene und komplexe Pflegesituationen und nicht Krankheitsbilder im Mittelpunkt pflegerischen Handelns stehen. Dabei werden ungedeutete Pflegephänomene, wie z. B. Unruhe, Schmerzen, muskuläre Verspannungen, Atemnot oder mangelnde Ansprechbarkeit in einen medizinischen, persönlichen, institutionellen und gesellschaftlichen Kontext eingebettet. Die Fallsituationen sind umso komplexer, desto mehr Verbindungen zwischen den situativen Phänomenen, den Bedingungen und den Wissensbeständen der Lernenden hergestellt werden können [30]. Die sieben Bearbeitungsschritte können quasi als methodische Anleitung zur Deutung der Situation aufgefasst werden.

Die Erweiterung des Deutungswissens bzw. die Generierung von bisher nicht mit dem Problem in Verbindung gebrachten Hypothesen wird beim problemorientierten Lernen angeregt

- durch die Verknüpfung der im Fall ermittelten Probleme mit den Kontextbedingungen und dem eigenen Vorwissen,

- durch die Konfrontation mit divergierenden Deutungen der anderen Gruppenteilnehmer,

- durch Impulse der Tutoren, die diese Deutungen hinterfragen, Feed-back geben oder Deutungsdifferenzen zur Sprache bringen.

\section{Ergebnisse der Evaluation}

Die Evaluation richtet sich sowohl auf die Struktur, den Prozess und das Ergebnis. Es handelt sich um eine formative Evaluation, im Vordergrund steht also die Ermittlung von Verbesserungsmöglichkeiten im konkreten Projekt. Da die potenziellen Probleme, die Wirkungen und die Verbesserungsmöglichkeiten zunächst als unbekannt angesehen werden müssen, ist von der Offenheit des Forschungsgegenstands auszugehen. Infolgedessen werden vorrangig qualitative Methoden der Datenerhebung und -auswertung, wie qualitative Interviews und unstrukturierte Formen der Beobachtung eingesetzt [31]. Quantitative Verfahren werden dann ver- wendet, wenn sich bei den vorhergehenden Untersuchungen bereits Kategorien herauskristallisiert haben oder theoretisch gewonnene Kategorien in Bezug auf Probleme oder Wirkungen überprüft werden sollen. Im Folgenden werden ausgewählte Ergebnisse der Ergebnisevaluation anhand von Teilnehmerbefragungen nach einem Jahr vorgestellt. Die Ergebnisevaluation erfolgt zum einen unter der Maßgabe der Teilnehmerzufriedenheit und zum anderen unter der Maßgabe didaktischer Kriterien.

Aus Teilnehmersicht bestehen Kriterien einer erfolgreichen Fachweiterbildung im Wissenserwerb, in der Erlangung von Souveränität im beruflichen Alltag und in der Befähigung zur Verbesserung der Praxis ${ }^{1}$. In einer Fragebogenerhebung nach einem Jahr machten zwei Drittel der Teilnehmer auf die Frage: „Was förderte die Zielerreichung“ (im Sinne der Teilnehmer) beim klinischen Unterricht und beim medizinischen Dozentenunterricht die Angabe „stimmt voll und ganz“ oder „stimmt größtenteils“. Den Dozentenunterricht im Fach Pflege schätzten dagegen mehr als 50\% der Teilnehmer als lediglich mittelmäßig wichtig für die Zielerreichung ein [32]. Beim Problemorientierten Lernen wurden alle fünf Antwortalternativen angekreuzt, Häufungen gab es im mittleren Bereich.

Die reflexive Wissensverwendung bei der Deutung von Situationen aus der Intensivpflege ist ein zentrales Ziel aus didaktischer Sicht. Da bislang in erster Linie das Problemorientierte Lernen in diese Richtung weiterentwickelt wurde, beziehen sich die folgenden Ergebnisse ausschließlich darauf. In qualitativen Interviews nach einem Jahr lassen die Äußerungen einiger Teilnehmer auf einen positiven Effekt des Problemorientierten Lernens schließen:

- „Gibt viele Denkanstöße. Gibt oft eine Änderung der Denkweise, man wird offener."

- „Macht offen durch das Hinterfragen“.

- „Am Anfang war das Reflektieren schwierig, aber man lernt dazu.“

- „Auch am Patienten kann ich POL gut umsetzen: Was sehe ich? Was kann ich machen? Ich gehe Schritt für Schritt vor.“

Kurs 01/03 im Januar 2003 
Die ersten drei Äußerungen sprechen dafür, dass die Teilnehmer eine stärker kritische und hinterfragende Haltung dem eigenen Urteil gegenüber aufgebaut haben. Die letzte Äußerung kann als Umsetzung der prozessorientierten Pflege interpretiert werden.

In den gleichen qualitativen Interviews negieren andere Teilnehmer diesen Effekt und hinterfragen zudem die Relevanz der Ziele:

- „POL und Station sind für mich ein absoluter Unterschied. Es ist praktisch nicht möglich, das anzuwenden, was theoretisch vermittelt wird. Die Zeit ist zu knapp.“

- „Ich arbeite und handle aus Erfahrung und fange nicht an, Hypothesen zu bilden. Das weiß man.“

- „Es reicht doch, wenn man mit dem Patienten spricht, sich seine Akte ansieht oder eine Kollegin fragt. Ich mag dieses ganze Hypothesengetue nicht."

- „Am Bett kann man ganz anders reagieren. Das hat nichts damit $\mathrm{zu}$ tun, dass die Fälle vielleicht $\mathrm{zu}$ schwierig sind.“

- „Ich hätte lieber guten Dozentenunterricht.“

Diese Äußerungen können als Ablehnung sowohl des reflektierenden Denkens als auch des Pflegeprozessgedankens gedeutet werden und belegen die Beibehaltung des verrichtungsorientierten, mechanistischen Pflegehandelns.

Betrachtet man die Ergebnisse resümierend, so lässt sich erstens feststellen, dass die meisten Teilnehmer die Wirksamkeit der traditionellen Lehr-Lernformen höher einschätzen als die der innovativen Ansätze. Dies könnte möglicherweise damit zusammenhängen, dass den Teilnehmern aufgrund ihrer durch abhängiges Lernen geprägten Sozialisation in der allgemeinbildenden Schule und in der Krankenpflegeausbildung das selbstverantwortliche Lernen fremd ist.

Zweitens weisen die Äußerungen der Teilnehmer darauf hin, dass der aus didaktischer Sicht angestrebte Lerneffekt, also die Erweiterung der Deutungs- und Reflexionskompetenz, zumindest bei einigen Teilnehmern eingetreten ist. Andere dagegen negieren den Effekt nicht nur, sondern werten sogar die gesamte Intention ab. Verantwortlich hierfür könnte zum einen das in der Praxis vorherrschende mechanistische und verrichtungsorientierte Pflegeverständnis sein, d.h. vom Funktionsfeld wird die Reflexionskompetenz der Teilnehmer nicht verlangt. Außerdem darf nicht übersehen werden, dass die geforderte reflexive Haltung für die Teilnehmer eine Verunsicherung darstellt, da bisher geltende Gewissheiten infrage gestellt werden. Dies widerspricht dem Ziel der Souveränität und Handlungssicherheit der Teilnehmer. Schließlich ist möglicherweise auch die Durchführung der Methode des Problemorientierten Lernens noch verbesserungsbedürftig.

Diese Befunde sind insbesondere für die betroffenen Lehrer zunächst entmutigend. Zugleich können sie aber auch als Ansporn aufgefasst werden, curriculare Reformen in der Pflegeaus-, -fort- und -weiterbildung noch stärker als bisher voranzutreiben. Dabei dürfen Innovationen nicht allein Bildungsmaßnahmen überlassen bleiben, vielmehr muss sich auch das Funktionsfeld ändern, wenn die Bildungsreformen wirksam sein sollen.

\section{Literatur}

${ }^{1}$ Justizbehörde der Freien und Hansestadt Hamburg. Amtlicher Anzeiger Teil III des Hamburgischen Gesetz- und Verordnungsblattes; Fortbildungs- und Prüfungsordnung zur Fachkrankenschwester/zum Fachkrankenpfleger und zur Fachkinderkrankenschwester/zum Fachkinderkrankenpfleger für Intensivpflege und Anästhesie. 2000

2 Benner P. Stufen zur Pflegekompetenz. From novice to expert Bern: Huber, 1994

${ }^{3}$ Blumer H. Der methodologische Standpunkt des symbolischen Interaktionismus. In: Arbeitsgruppe Bielefelder Soziologen (Hrsg). Alltagswissen, Interaktion und gesellschaftliche Wirklichkeit. Band I Reinbek: Rowohlt, 1973: 80-146

${ }^{4}$ Oevermann U. Theoretische Skizze einer revidierten Theorie professionalisierten Handelns. In: Combe A, Helsper W (Hrsg). Pädagogische Professionalität Frankfurt/ Main: Suhrkamp, 1996: 70-182

${ }^{5}$ Neuweg GH. Könnerschaft und implizites Wissen. Münster: Waxmann, 1999

${ }^{6}$ Oevermann U. Theoretische Skizze einer revidierten Theorie professionalisierten Handelns. In: Combe A, Helsper W (Hrsg). Pädagogische Professionalität. Frankfurt/ Main: Suhrkamp, 1996: 70-182

${ }^{7}$ Neuweg GH. Könnerschaft und implizites Wissen. Münster: Waxmann, 1999
${ }^{8}$ Dewe B. Wissensverwendung in der Fort- und Weiterbildung. Baden-Baden: Nomos, 1988

${ }^{9}$ Pätzold G. Lernortkooperation im Lernfeldkonzept. www.PR-INTERNET.com für die Pflege 2004; 6: 1: 5-13

${ }^{10}$ Robinsohn SB. Bildungsreform als Revision des Curriculums. Neuwied, Berlin: Luchterhand, 1969

${ }^{11}$ Lipsmeier A, Pätzold G (Hrsg). Lernfeldorientierung in Theorie und Praxis. Beiheft 15 der ZBW Stuttgart: Steiner, 2000

${ }^{12}$ Greb U. Ein Strukturgitter für die Fachweiterbildung Intensivpflege. www.PRINTERNET.com für die Pflege 2001; 3: 11: 252-269

${ }^{13}$ Dörig R. Das Konzept der Schlüsselqualifikationen. Ansätze, Kritik und konstruktivistische Neuorientierung auf Basis der Erkenntnisse der Wissenspsychologie. Hallstadt: Rosch-Buch, 1994

${ }^{14}$ Zimmermann M. Transferfördernde Berufserziehung in Schule und Betrieb. Zum „Expertenkulturansatz“ in der Didaktik der kaufmännischen Berufserziehung. In: Beck K, Müller W, Deißinger T, Zimmermann M (Hrsg). Berufserziehung im Umbruch Weinheim: Deutscher Studienverlag, 1996: 45-60

${ }^{15}$ Mandl H, Prenzel M, Gräsel C. Das Problem des Lerntransfers in der betrieblichen Weiterbildung. Unterrichtswissenschaft 1992; 20, 2: 126-143

${ }^{16}$ Reetz L. Wissen und Handeln. - Zur Bedeutung konstruktivistischer Lernbedingungen in der kaufmännischen Berufsbildung. In: Beck K, Müller W, Deißinger T, Zimmermann M (Hrsg). Berufserziehung im Umbruch Weinheim: Deutscher Studienverlag, 1996: 173-188

${ }^{17}$ Neuweg GH. Könnerschaft und implizites Wissen. Münster: Waxmann, 1999

${ }^{18}$ Bögemann-Großheim E, Brendel $S$, Handgraaf M. Problem-based Learning eine pädagogische Antwort auf neue Herausforderungen in der Krankenpflege. PflegePädagogik 1999; 2: 4-11

${ }^{19}$ Meer Kvan. Problemorientiertes Lernen. In: Schwarz-Govaers R (Hrsg). Standortbestimmung Pflegedidaktik. Referate zum 1. Internationalen Kongress zur Didaktik der Pflege Aarau: Verlag der Kaderschule für die Krankenpflege, 1994: 81-93

${ }^{20}$ Moust JHC, Bouhuis PAJ, Schmidt HG. Problemorientiertes Lernen. Wiesbaden: Ullstein Medical, 1999

${ }^{21}$ Schwarz-Govaers R. Problemorientiertes Lernen in der Pflegeausbildung. www. PR-INTERNET.com für die Pflege 2002; 4: $2: 30-45$

22 Schwarz-Govaers R. Problemorientiertes Lernen - neuer Wein in alten Schläuchen oder eher alter Wein in neuen Schläuchen? www.PR-INTERNET.com für die Pflege 2003; 5: 1: 36-45 
${ }^{23}$ Boonen A, Darmann I. Neue Lehr-/Lernformen in der Fachweiterbildung Anästhesie- und Intensivpflege. Zwischenbericht. Teil I: Konzeption (Boonen). Teil II: Evaluation (Darmann). Unveröffentlichtes Manuskript. Hamburg/Bremen, 2003

${ }^{24}$ Brinker-Meyendriesch E. Evidenzbasierung: Wissen, Handeln und Lernen in der Pflege. Pflege 2003; 16 (4): 230-235

${ }^{25}$ Werbke SR. Problemorientiertes Lernen als Grundlage für eine Evidenzbasierte Praxis. www.PR-INTERNET.com für die Pflege 2001; 3: 9: 166-171

${ }^{26}$ van Meer K. Problemorientiertes Lernen. In: Schwarz-Govaers R (Hrsg). Standortbestimmung Pflegedidaktik. Referate zum 1. Internationalen Kongress zur Didaktik der Pflege Aarau: Verlag der Kaderschule für die Krankenpflege, 1994: 81-93

${ }^{27}$ Moust JHC, Bouhuis PAJ, Schmidt HG. Problemorientiertes Lernen. Wiesbaden: Ullstein Medical, 1999

${ }^{28}$ Schwarz-Govaers R. Problemorientiertes Lernen in der Pflegeausbildung. www. PR-INTERNET.com für die Pflege 2002; 4: $2: 30-45$

${ }^{29}$ Schwarz-Govaers R. Problemorientiertes Lernen - neuer Wein in alten Schläuchen oder eher alter Wein in neuen Schläuchen? www.PR-INTERNET.com für die Pflege 2003; 5: 1: 36-45

${ }^{30}$ Darmann I. Problemorientiertes Lernen Transfer durch die Erweiterung von Situationsdeutungen. www.PR-INTERNET.com für die Pflege 2004 (im Druck)

${ }^{31}$ Lamnek S. Qualitative Sozialforschung. Band II: Methoden und Technik. Weinheim: Beltz, PsychologieVerlagsUnion, 1993

32 Boonen A, Darmann I. Neue Lehr-/Lernformen in der Fachweiterbildung Anästhesie- und Intensivpflege. Zwischenbericht. Teil I: Konzeption (Boonen). Teil II: Evaluation (Darmann). Unveröffentlichtes Manuskript. Hamburg/Bremen, 2003

\section{Prof. Dr. Ingrid Darmann}

Universität Bremen, Fachbereich Human- und Gesundheitswissenschaften Grazer Str. 6 28359 Bremen

E-mail: darmann@uni-bremen.de 